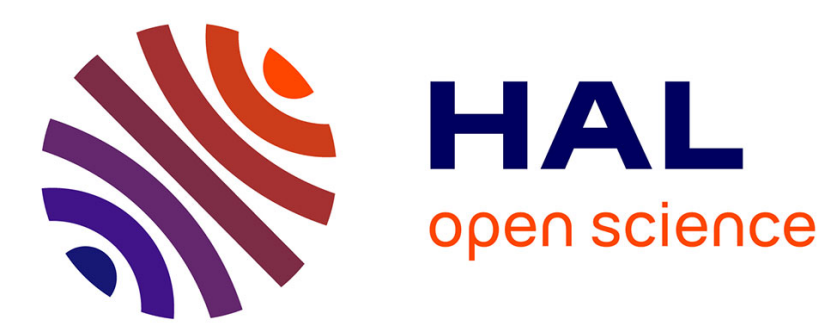

\title{
DIVISION CONJUGALE DU TRAVAIL ET LÉGITIMITÉ PROFESSIONNELLE
}

\author{
Christophe Giraud, Jacques Rémy
}

\section{To cite this version:}

Christophe Giraud, Jacques Rémy. DIVISION CONJUGALE DU TRAVAIL ET LÉGITIMITÉ PROFESSIONNELLE: Le cas des activités de diversification agricole en France. Travail, genre et sociétés, 2013, 30, pp.155-172. 10.3917/tgs.030.0155 . hal-01448716

\section{HAL Id: hal-01448716 \\ https://hal-univ-paris.archives-ouvertes.fr/hal-01448716}

Submitted on 28 Jan 2017

HAL is a multi-disciplinary open access archive for the deposit and dissemination of scientific research documents, whether they are published or not. The documents may come from teaching and research institutions in France or abroad, or from public or private research centers.
L'archive ouverte pluridisciplinaire HAL, est destinée au dépôt et à la diffusion de documents scientifiques de niveau recherche, publiés ou non, émanant des établissements d'enseignement et de recherche français ou étrangers, des laboratoires publics ou privés. 


\title{
DIVISION CONJUGALE DU TRAVAIL ET LÉGITIMITÉ PROFESSIONNELLE
}

Le cas des activités de diversification agricole en France

Christophe Giraud et Jacques Rémy

\section{La Découverte | Travail, genre et sociétés}

\author{
2013/2 - $n^{\circ} 30$ \\ pages 155 à 172
}

ISSN 1294-6303

Article disponible en ligne à l'adresse:

http://www.cairn.info/revue-travail-genre-et-societes-2013-2-page-155.htm

Pour citer cet article

Giraud Christophe et Rémy Jacques, « Division conjugale du travail et légitimité professionnelle » Le cas des activités de diversification agricole en France,

Travail, genre et sociétés, 2013/2 n³0, p. 155-172. DOI : 10.3917/tgs.030.0155

Distribution électronique Cairn.info pour La Découverte.

(c) La Découverte. Tous droits réservés pour tous pays.

La reproduction ou représentation de cet article, notamment par photocopie, n'est autorisée que dans les limites des conditions générales d'utilisation du site ou, le cas échéant, des conditions générales de la licence souscrite par votre établissement. Toute autre reproduction ou représentation, en tout ou partie, sous quelque forme et de quelque manière que ce soit, est interdite sauf accord préalable et écrit de l'éditeur, en dehors des cas prévus par la législation en vigueur en France. II est précisé que son stockage dans une base de données est également interdit. 


\section{DIVISION CONUUGALE DU TRAVAIL ET LÉGITIMITÉ PROFESSIONNELLE}

Christophe Giraud et Jacques Rémy

$\mathrm{L}$

e modèle de l'agriculteur professionnel, initié par les lois d'orientation agricoles du début des années 1960 et par la mise en œuvre de tout un package technico-économique accompagné d'un discours idéologique fort, a durablement imposé l'image idéale de l'agriculteur spécialisé qui développe un nombre limité de productions (végétales ou animales) dans la ligne de compétences acquises par une formation technique adaptée, et dont le métier est centré sur une seule activité, source des revenus de l'exploitation et de la famille ${ }^{1}$. Porté par les syndicats et par l'appareil d'encadrement agricole, ce modèle s'est substitué à celui de polyculture-élevage, tandis que les activités de transformation et de vente à la ferme étaient considérées comme archaïques par le discours professionnel dominant.

Dans cette ligne, l'appareil d'encadrement agricole a travaillé à définir les actes et activités qui faisaient légitimement partie du métier d'agriculteur et à favoriser les systèmes de production qui transformaient les agriculteurs en producteurs de matières premières pour les industries agroalimentaires ${ }^{2}$. Il a également promu un modèle d'exploitation agricole à deux unités de travail humain dont mari et épouse seraient les acteurs principaux. L'exploitation devait se confondre avec la famille conjugale et lui donner les moyens de vivre

${ }^{1}$ La loi 60-808 du 5 août 1960 a pour objet, selon l'article 2, alinéa 7 « de promouvoir et favoriser une structure d'exploitation de type familial, susceptible d'utiliser au mieux les méthodes techniques modernes de production et de permettre le plein emploi du travail et du capital d'exploitation ". Dans l'article 7, on évoque «la superficie que devrait normalement avoir une exploitation mise en valeur directement par deux unités de main-d'œuvre, ou plus en cas de société de culture ou de groupements d'exploitants » (JO du 7 août 1960, p. 7360 et p. 7361 )

${ }^{2}$ Voir note page suivante. 
${ }^{2}$ Les coopératives ont promu l'idée que la création de la valeur ajoutée devait se faire au sein de l'outil collectif, pour le bien commun. Le discours sur l'agriculteur spécialisé, professionnel, liait donc une certaine image de l'efficacité technique avec celle d'une solidarité, d'une collaboration au sein du groupe professionnel.

\footnotetext{
${ }^{3}$ Mentionnées pour mémoire, ces activités ne font pas ici l'objet d'une analyse spécifique.

${ }^{4}$ Depuis la loi de modernisation de 1988 mais seulement dans la mesure où ces activités s'appuient sur les facteurs de production de l'exploitation et où elles ne dépassent pas une part donnée du chiffre d'affaires global. Ce n'est pas le cas dans les pays anglo-saxons où ces activités ne sont pas considérées comme agricoles.
}

dignement, afin d'atteindre la parité avec les autres groupes sociaux. L'activité professionnelle à l'extérieur de l'exploitation, du mari comme de la femme, a ainsi été vivement critiquée et combattue [Rémy, 1987]. Suivant le Code civil, c'était le mari qui, pendant longtemps, avait la seule responsabilité de l'exploitation et le seul statut professionnel, chef d'exploitation, condamnant les femmes à la situation d'aides familiales, c'est-à-dire de travailleuses à titre gratuit. Cette situation les rendait particulièrement dépendantes de leur mari [Barthez, 1982 ; Delphy, 1983 ; Lagrave, 1987] et les cantonnait souvent dans des activités peu valorisantes (travail d'astreinte, gestion des papiers) ou des rôles de bouche-trou [Berlan, 1982], c'est-à-dire les positions les moins bien considérées dans la division du travail sur l'exploitation. Et, bien sûr, elles étaient assujetties aux rôles domestiques. L'organisation de l'exploitation agricole conjugale s'appuyait donc sur des stéréotypes de sexe très clivants. Cependant, l'action des conseillères ménagères et agricoles auprès des groupements féminins agricoles a contribué à l'acquisition de nouvelles compétences et à l'ouverture au monde extérieur des exploitantes engagées dans le mouvement dit de développement agricole [Dentzer, 1974].

Ainsi, en dépit des incitations fortes portées par le modèle d'intensification et de spécialisation, de nouvelles activités sont peu à peu apparues ou (re)devenues visibles dans le monde agricole. L'hébergement touristique, la transformation de produits fermiers, la vente directe, les activités agri-environnementales ${ }^{3}$, progressivement reconnus comme agricoles ${ }^{4}$, font de plus en plus du métier d'agriculteur une combinaison d'activités, notamment marchandes. La montée de ces nouvelles activités a souvent été associée aux nouvelles contraintes économiques qui pèsent sur les exploitations agricoles et comme une manière de "s'en sortir» économiquement («coping strategies») [Muller, Faure et Gerbaux, 1989; Marsden et al., 1989].

Le travail régulateur des organisations agricoles n'a pas $\mathrm{pu}$, non plus, supprimer les exploitations pluriactives et, à partir des années 1980, une part croissante des femmes d'agriculteur se sont massivement tournées vers le marché du travail salarié non agricole. En 2005, après pourtant de nombreuses modifications législatives améliorant le statut des femmes en agriculture, «sur cent jeunes conjointes d'agriculteurs, environ soixante exercent une profession hors de la ferme à laquelle elles n'apportent aucun travail. Dix autres travaillent à la fois à l'extérieur et sur l'exploitation à titre secondaire. Vingt autres se consacrent exclusivement à l'exploitation et les dix dernières sont sans profession, femmes au foyer. Ce sont les conjointes les plus âgées qui travaillent principalement sur l'exploitation » [Rattin, 2006, p. 18]. 
L'éclosion, au sein des exploitations agricoles, de nouvelles activités économiques comme le tourisme (chambres d'hôtes, gîtes ruraux, campings à la ferme, ferme-auberge...) a pu être interprétée comme une nouvelle opportunité offerte aux femmes restées sur l'exploitation agricole pour modifier la division sexuée du travail et les rapports sociaux de sexe. En effet, plusieurs auteurs (par exemple Gemma Cánoves, María-Dolores García-Ramón et Nuria Valdovinos [1993] ou Mary Bouquet [1985]) ont insisté sur le rôle déterminant des femmes dans ce type d'activité en raison d'une proximité avec leurs rôles domestiques.

La question que nous poserons donc est celle de l'articulation entre le développement de ces nouvelles activités très variées en agriculture, les logiques professionnelles qui définissent le métier d'agriculteur ainsi que la répartition des rôles féminins et masculins qui se fait en lien avec les stéréotypes de sexe. Ces nouvelles activités agricoles constituentelles de nouvelles opportunités pour les femmes restées sur les exploitations agricoles?

\section{RECOMPOSITION DU MÉTIER D'AGRICULTEUR ET DIVISION CONJUGALE DU TRAVAIL SUR L'EXPLOITATION}

Cette multiplication de nouvelles combinaisons d'activités au sein des exploitations agricoles a été désignée, non sans flottement, par le terme de «diversification ». Notre usage du terme est restrictif : la diversification nous intéresse dans la mesure où, sur l'exploitation, de nouvelles activités se combinent avec les activités agricoles reconnues légitimes par la profession. Les travaux anglo-saxons (depuis Brian Ilbery [1991]) distinguent une "diversification agricole", qui renvoie à la mise en culture ou à l'élevage de produits nouveaux dans une zone géographique donnée, et une «diversification structurelle", qui fait référence au développement de nouveaux services à valeur ajoutée qui ont une forte dimension commerciale. C'est cette seconde forme de diversification qui nous intéresse ici.

\section{La diversification, une notion polysémique}

La diversification structurelle, approchée par l'appareil statistique français en 2000, comprend :

* la vente directe de produits fermiers (de vin, de fromage...) adoptée par 15,4\% des exploitations en 2000, ou la vente d'énergie renouvelable (énergie éolienne, combustion de paille...) ;

* la transformation de produits de la ferme pour la vente, produits laitiers, charcuteries... (effectuée par 9,2\% des exploitations en 2000), ou du bois de l'exploitation (sciage, bois de chauffage...);

* l'hébergement à la ferme (gîtes ou chambres d'hôtes), la restauration (ferme-auberge ou table d'hôte) réalisée par 1,9\% des exploitations en 
${ }^{5}$ Dix ans plus tard, en 2010, ce taux aurait régressé à $12 \%$ [Lerbourg, 2013] mais les activités de diversification mesurées en 2010 ne sont pas les mêmes elles ne comportent pas la vente directe qui était l'activité la plus fréquente en 2000 et 1988.
2000 (proportion stable en 2010), les autres activités récréatives et l'artisanat ;

* les travaux à façon (travaux agricoles payants effectués pour d'autres exploitations, proposés par 2,1\% des exploitations agricoles en 2000 et $3 \%$ en 2010, proportion dépassant ainsi nettement en nombre celle des activités touristiques), les travaux de sylviculture, d'exploitation forestière ou de scierie, d'entretien de paysage, déblaiement de neige... et autres travaux agricoles.

En revanche les labels ou les signes de qualité constituent des «produits agricoles » spécifiques développés par les exploitations (diversification agricole) et non pas de nouveaux services (diversification structurelle). Ils ne sont donc pas pris en compte ici.

Les diverses activités relevant du processus de diversification structurelle seront nommées « activités diversifiées ».

Donnons tout d'abord quelques repères statistiques: en 2000 , presque un quart des exploitations françaises $(22,7 \%)$ ont au moins une activité diversifiée ${ }^{5}$. Ce taux a globalement progressé en France, depuis les années 1980, mais de manière assez différente selon les activités. La diversification, généralement considérée comme une réponse à la crise de surproduction des années 1970, a été mise en rapport avec des déterminants économiques et géographiques. Ruth Gasson [1988] et Brian Ilbery [1991] ont montré que la taille de l'exploitation avait un impact positif sur le développement des activités diversifiées. Les exploitations les plus importantes peuvent mieux mettre à profit les aides à la diversification qui existent au Royaume-Uni. De même, la proximité du marché urbain a un impact positif sur la vente directe ou la transformation de produits et négatif sur le tourisme et les activités récréatives. En France, Danièle Capt et Anne-Marie Dussol [2004] ont montré que la taille de l'exploitation avait plutôt un impact négatif sur les activités de vente directe ou de transformation et positif pour les activités de travail à façon. Les données du recensement agricole 2010 confirment que les exploitations diversifiées dans leur ensemble sont surreprésentées parmi les grandes exploitations (et sous-représentées parmi les petites exploitations). Les travaux à façon en sont la raison : $60 \%$ des exploitations réalisant des travaux à façon se situent parmi les grandes exploitations alors qu'elles ne représentent qu'un tiers de l'ensemble des exploitations agricoles françaises. Ces mêmes exploitations effectuant des travaux à façon sont en revanche sous-représentées parmi les exploitations moyennes ou petites. En 2010, les activités touristiques et de transformation sont en revanche légèrement surreprésentées dans les exploitations de dimension économique moyenne [Lerbourg, 2013, p. 2]. La diversification varie également selon le secteur de production : la vente directe est très pratiquée dans l'horticulture ou le maraîchage, l'accueil touristique dans les exploitations spécialisées en élevage ovin ou caprin (souvent situées en montagne), le travail à façon dans les exploitations céréalières et horticoles (qui ont un parc de matériel à rentabiliser). 
Une grande partie de ces auteurs considère que le choix d'une activité de diversification se fait essentiellement en fonction des ressources disponibles sur l'exploitation et que les acteurs économiques peuvent s'adapter et mettre en place avec une certaine souplesse une nouvelle activité économique. Nous sommes sensibles, en revanche, à la force de l'identité professionnelle et aux difficultés d'adaptation des acteurs et des institutions à une agriculture diversifiée [Lémery, 2003 ; Rémy, 1987]. Cette dimension professionnelle, qui agit parallèlement aux facteurs macroéconomiques nous intéressera plus particulièrement dans cet article.

Chaque métier construit un discours qui définit les tâches et activités qui sont centrales, celles qui ont un caractère secondaire [Hughes, 1996] et celles qui n'en font pas partie. Les activités diversifiées restent, du point de vue du discours professionnel dominant, des activités agricoles de seconde zone. Ces écarts n'ont toutefois pas la même portée selon les activités et le secteur (et probablement la région puisque le discours professionnel est aussi fonction de l'histoire syndicale locale). L'agriculture est en effet la réunion plus ou moins hétérogène de secteurs de production assez différenciés qui peuvent être considérés comme autant de métiers différents. Chacun de ces secteurs construit un discours professionnel qui définit les activités faisant partie du métier et celles qui lui sont extérieures, probablement en raison de l'existence d'appareils d'encadrement spécifiques à chaque secteur [Rémy, 1987].

La notion de multifonctionnalité de l'agriculture, introduite en France depuis quelques années, vise cependant à légitimer les différentes fonctions de l'activité agricole et à donner aux nouvelles missions de ce secteur une dignité égale à la fonction de production, dans la perspective d'un développement durable désormais soutenu et porté par le monde politique. Cependant, la fonction sociale comme la fonction environnementale, si elles sont aujourd'hui bien présentes dans le discours des responsables professionnels les plus lucides, ne sont pas considérées par les agriculteurs, sauf exception, comme aussi vitales pour la société et pour eux-mêmes que la fonction productive. Celle-ci, avec les aides qui lui ont été longtemps directement associées (et sont aujourd'hui partiellement « découplées »), demeure leur principale source de revenus et conserve ainsi la prééminence sur les autres fonctions.

Comment les agriculteurs qui ont choisi la voie de la diversification structurelle ou celle de la multifonctionnalité gèrent-ils cette tension entre des activités principales, prestigieuses, et les autres activités (secondaires ou extérieures), moins bien considérées ? En suivant Everett C. Hughes [1996], nous faisons l'hypothèse que cette tension au sein de l'identité professionnelle des agriculteurs se résout par une division du

\footnotetext{
${ }^{6}$ Le maraîchage est l'un de ceux où le modèle de pratique est le plus éloigné du discours dominant car il continue à inclure parmi les actes légitimes celui de la vente directe des produits au consommateur (le développement rapide des associations pour le maintien d'une agriculture paysanne - AMAP - qui organisent un système d'achat régulier de paniers de produits et de contacts directs entre les producteurs et les consommateurs, tend à renouveler et élargir cette pratique).
} 
${ }^{7}$ La loi du 5 janvier 2006 a franchi un pas vers une meilleure intégration des conjointes, notamment en termes de protection sociale et de retraite, mais aussi d'accession à une réelle reconnaissance professionnelle.

${ }^{8}$ L'apport des autres membres de la famille au travail de l'exploitation ne sera pas examiné dans cet article. travail particulière. Les tâches les moins prestigieuses ont tendance à être déléguées à d'autres personnes qui gravitent autour des "professionnels". Tout comme dans le monde salarié, l'exploitation agricole serait le lieu d'une délégation des tâches considérées comme subalternes aux collaborateurs moins bien placés : conjoint, ascendants ou enfants, salariés.

En raison d'une division sexuée du travail et avec des différences de statut très marquées [Barthez, 1982; Lagrave, $1^{1987]^{7}}$, les activités diversifiées auraient donc plus tendance à être réalisées par les conjointes que par les maris. Cette division du travail permettrait au chef d'exploitation de ne pas trop se différencier de ses pairs malgré l'écart à la norme de la profession que constitue le développement d'une activité diverse sur l'exploitation. La question de la répartition conjugale du travail dans l'exploitation ${ }^{8}$ et des stéréotypes de sexe, nous paraît constituer une des clés de la consolidation et de la réaffirmation des identités professionnelles au travers (et en dépit) des recompositions des métiers d'agriculteur et d'éleveur. C'est cette hypothèse que nous allons chercher à vérifier.

\section{LA PLACE DES FEMMES DANS LES ACTIVITÉS DE DIVERSIFICATION}

Nous traiterons tout d'abord de la corrélation de la diversification structurelle avec la situation conjugale du chef d'exploitation, puis avec l'engagement de la conjointe sur l'exploitation, enfin avec le partage conjugal du travail lié aux activités diversifiées.

Des données tirées des recensements agricoles de 1988 et 2000

Nous nous appuyons sur deux bases de données différentes. La pre-

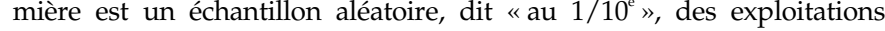
françaises, constitué à partir du recensement agricole réalisé en 2000 par le ministère de l'Agriculture, soit 65593 exploitations. La seconde est un échantillon aléatoire, dit " au 1/10" », de 71011 exploitations, construit à partir du recensement général de l'agriculture de 1988.

Nous nous limiterons à l'ensemble des exploitations individuelles conduites par un homme ou des exploitations en société dont l'homme et éventuellement sa conjointe peuvent avoir des statuts équivalents (51 150 en 2000 et 62598 en 1988 dans nos échantillons au 1/10 ${ }^{\circ}$ ) et nous laisserons de côté celles conduites exclusivement par les femmes. Certes, ces dernières constituent une frange désormais importante des exploitations agricoles françaises qui ne peut être négligée et traduit les transformations de la place des femmes dans le milieu agricole [Dahache, 2010]. Les exploitations où les femmes ont un statut de cheffe d'exploitation ou d'associée représentent $23 \%$ des exploitations agricoles en France en 2004. Cependant, les exploitations féminines sont hétérogènes: une part de celles-ci $(17 \%$ des chefs d'exploitation féminins, en 2009, selon la Mutualité sociale agricole) est conduite formellement par les femmes au moment où le mari part en retraite, mais ces exploitations continuent à être dirigées de fait par le retraité. L'autre part correspond à un véritable contrôle des femmes sur l'exploitation. Dans ce cas précis, les conjoints masculins ont presque

$160 \Delta$ Travail, genre et sociétés $n^{\circ} 30$ - Novembre 2013 
Division conjugale du travail et légitimité professionnelle

tous une activité professionnelle extérieure à l'exploitation. Très peu sont aides familiaux. La femme se retrouve donc conjugalement seule responsable sur l'exploitation (et c'est elle qui assurera alors le travail agricole comme para-agricole). La question du partage du travail agricole et para-agricole se pose donc de façon plus aiguë pour les exploitations agricoles conduites par un homme ou conjointement par un homme et une femme, c'est pour cette raison que nous nous limitons à cette souspopulation.

\section{Deux types d'activités diversifiées}

Dans quelle mesure l'adoption d'une activité diversifiée par les exploitations agricoles dirigées par un homme est-elle liée à l'engagement professionnel des compagnes dans ces exploitations?

Si l'on met de côté les exploitations où le chef n'a pas ou plus de conjointe, soit $27,8 \%$ des exploitations en 2000, on peut distinguer cinq catégories d'engagement de la compagne qui définissent autant de types d'exploitation:

- il y a celles où elle ne travaille qu'en dehors de l'exploitation sans participer au travail agricole, soit $22,7 \%$ des exploitations ;

- celles où elle fait les deux, soit $8,5 \%$ des cas ;

- celles où elle n'a pas d'activité professionnelle hors de l'exploitation et n'est pas active non plus sur l'exploitation, soit $17,3 \%$;

- celles où elle travaille uniquement sur l'exploitation mais moins d'un mi-temps, soit 9,3\% ;

- et celles où elle travaille uniquement sur l'exploitation et pour plus d'un mi-temps, soit $14,3 \%$ des cas.

Tableau 1 : Taux d'activités diversifiées et engagement professionnel de la conjointe en 2000 et 1988

\begin{tabular}{|c|c|c|c|c|c|c|c|}
\hline & \multicolumn{2}{|c|}{ Vente directe } & \multirow{2}{*}{$\frac{\text { Transformation }}{2000}$} & \multicolumn{2}{|c|}{ Hébergement } & \multicolumn{2}{|c|}{ Travail à façon } \\
\hline & 1988 & 2000 & & 1988 & 2000 & 1988 & 2000 \\
\hline Pas de conjointe & $19,3 \%$ & $12,7 \%$ & $7,9 \%$ & $1,3 \%$ & $1,1 \%$ & $1,5 \%$ & $2,1 \%$ \\
\hline $\begin{array}{l}\text { Conjointe active } \\
\text { seulement hors } \\
\text { exploit. }\end{array}$ & $16,3 \%$ & $13,4 \%$ & $7,9 \%$ & $2,0 \%$ & $1,6 \%$ & $2,7 \%$ & $3,3 \%$ \\
\hline $\begin{array}{l}\text { Conjointe active } \\
\text { sur et hors exploit. }\end{array}$ & $21,7 \%$ & $20,8 \%$ & $13,1 \%$ & $4,4 \%$ & $2,8 \%$ & $3,3 \%$ & $3,6 \%$ \\
\hline Conjointe inactive & $19,3 \%$ & $13,7 \%$ & $7,2 \%$ & $1,6 \%$ & $1,1 \%$ & $1,6 \%$ & $1,4 \%$ \\
\hline $\begin{array}{l}\text { Conjointe active } \\
\text { seulement sur } \\
\text { l'exploit. mais moins } \\
\text { d'un mi-temps }\end{array}$ & $22,4 \%$ & $16,4 \%$ & $9,0 \%$ & $2,3 \%$ & $2,4 \%$ & $2,7 \%$ & $2,4 \%$ \\
\hline
\end{tabular}


Christophe Giraud et Jacques Rémy

\begin{tabular}{ccc|c|cc|cc}
\hline $\begin{array}{c}\text { Conjointe active } \\
\text { sur 1'exploit. } \\
\text { pour un mi-temps } \\
\text { et plus }\end{array}$ & $22,3 \%$ & $21,6 \%$ & $13,6 \%$ & $1,8 \%$ & $2,7 \%$ & $1,9 \%$ & $2,4 \%$ \\
\hline $\begin{array}{c}\text { Toutes } \\
\text { exploitations }\end{array}$ & $20,6 \%$ & $15,5 \%$ & $9,3 \%$ & $1,9 \%$ & $1,7 \%$ & $2,1 \%$ & $2,5 \%$ \\
\hline
\end{tabular}

Source: Ministère de l'Agriculture, recensement agricole 2000, échantillon au $1 / 10^{\mathrm{e}}$, exploitation secondaire (en italique, chiffres du recensement général de l'agriculture 1988, échantillon au 1/10)

Champ : Exploitations avec chef masculin.

Lecture : En 2000, 12,7\% des chefs d'exploitation masculins qui ne vivent pas en couple développent une activité de vente directe.

Nous tirons de cette présentation un premier enseignement : la présence d'une conjointe dans le ménage (quelle que soit son activité professionnelle) semble constituer, pour un chef d'exploitation, une ressource qui favorise le développement d'activités diversifiées (en 2000, 12,7\% des exploitations sans conjointe ont une activité de vente directe contre $15,5 \%$ de toutes les exploitations). Cette présence de la conjointe est positive pour toutes les activités de diversification, même si son impact est plus ou moins fort selon l'activité considérée.

Deuxième résultat marquant: selon le type d'engagement professionnel de la conjointe, certaines activités diversifiées ont plus de chances d'être développées sur l'exploitation. Deux types d'activités diversifiées peuvent ainsi être distingués :

- Le premier type (qui comprend la vente directe, l'hébergement ou la transformation de produits fermiers, la restauration...), est fortement corrélé au travail de la conjointe sur l'exploitation. Une participation agricole féminine, combinée à une activité professionnelle extérieure, peut également favoriser ces activités. En 2000, 21,6 \% des chefs d'exploitations dont les conjointes travaillent plus d'un mitemps, 20,8\% des chefs dont la conjointe est bi-active ont une activité de vente directe, contre seulement $13,4 \%$ de ceux dont la conjointe travaille seulement à l'extérieur. La bi-activité (travailler à l'extérieur et sur l'exploitation) est un facteur positif dans la mesure où ces activités ont souvent un caractère saisonnier (tourisme ou vente directe se font souvent l'été), elles peuvent donc être effectuées par des compagnes salariées durant leurs congés annuels.

- Le second type (travaux agricoles à façon c'est-à-dire les travaux agricoles payants réalisés pour d'autres exploitations, déblaiement de neige, travaux de sylviculture) est, au contraire, observé dans les exploitations où la conjointe est peu ou pas présente. En 1988, 1,9\% des exploitations où la conjointe travaille plus d'un mi-temps pratiquent le travail à façon, contre 3,3\% de celles où elle est bi-active et $2,7 \%$ de celles où la conjointe travaille seulement à

$162 \Delta$ Travail, genre et sociétés n 30 - Novembre 2013 
Division conjugale du travail et légitimité professionnelle

l'extérieur. Une exploitation où la conjointe participe fortement au travail agricole développe moins souvent une activité de travail agricole à façon qu'une où la femme participe peu.

Trois éléments permettent de comprendre cette distinction entre ces deux types de diversification : la nature des territoires de l'exploitation concernés et des ressources mobilisées par ces activités ; la nature des tâches à effectuer et la nature des jugements sur le travail.

Le premier type (tourisme, transformation, vente directe), associé à une forte implication de la conjointe sur l'exploitation, renvoie à des activités souvent proches de la résidence, espace plutôt féminin. Le second type (travaux à façon), où le rôle direct de la conjointe apparaît minime, est associé à l'utilisation des machines de l'exploitation et au travail dans les champs, domaine qui demeure encore plutôt masculin. La diversification respecte ainsi les stéréotypes de sexe.

En second lieu, les activités du premier type constituent un «faisceau de tâches" très différent de l'activité agricole: l'accueil touristique est l'exemple sans doute le plus évident d'un travail qui a pour support l'exploitation (et surtout la résidence) et dont les tâches concrètes (préparer le petitdéjeuner, refaire les lits, laver les draps, nettoyer) sont plus proches du travail ménager que du travail agricole [Giraud, 2001]. De même, la vente directe suppose la gestion d'une clientèle, tâche qui ne fait guère partie du travail agricole reconnu, sauf dans des secteurs bien déterminés de l'agriculture comme le maraîchage. Céline Bessière, dans son travail sur les exploitations viticoles de la région de Cognac, a montré comment la vente à la bouteille a pu constituer, pour certains viticulteurs, une échappatoire à la crise des débouchés et à la trop forte dépendance à l'égard des grandes maisons de négoce. Pourtant, les viticulteurs rencontrent des obstacles qui freinent ces velléités. Vendre le produit suppose de formuler un discours, sur le produit et ses qualités, et des compétences relationnelles qu'ils n'ont pas forcément. L'étude montre également que les réalisations qui ont été faites en ce domaine s'appuient bien souvent sur les femmes qui investissent du temps et mobilisent leur réseau relationnel [Bessière, 2006]. Marie-France Garcia-Parpet [2000] montre, chez les viticulteurs de la région de Chinon, que les conjointes peuvent apporter une aide très importante à la création d'une activité de vente directe de vin, notamment les femmes qui ne sont pas issues du milieu viticole local car elles peuvent donner accès à et solliciter un réseau de relations qui sont des clients potentiels. Le travail à façon, en revanche, mobilise en principe les mêmes compétences que celles que l'agriculteur engage dans sa propre exploitation. En dehors de la facturation, les tâches à réaliser sont essentiellement techniques et renvoient donc à la définition dominante de la profession. Il 
${ }^{9}$ Dans le recensement agricole 2000, la liste des activités est plus réduite. Les deux enquêtes ne sont donc pas tout à fait comparables.

${ }^{10}$ L'UTA ou « unité de travail annuel » correspond au temps de travail annuel légal cette convention ne correspond pas à un calcul véritable mais à des déclarations des chefs d'exploitation mises en forme par l'enquêteur.

L'évaluation semble avoir été souvent défavorable, dans le passé, à la prise en compte du travail féminin [Barthez, 1986 ; Lagrave, 1987].

${ }^{11}$ C'est par exemple le cas de formes anciennes de vente directe de surplus de production marchande ou

domestique, comme la vente de lait à la ferme pour les voisins proches ou encore le "panier de la fermière » vendu à quelques clients en ville [Houssel, 1970]. s'agit alors d'un élargissement de l'aire légitime de compétences et d'activités du chef d'exploitation. En outre, les clients sont tous des professionnels, des collègues, qui partagent le même langage et dont l'accès est plus aisé qu'un réseau de clients urbains de chambre d'hôtes.

Enfin, les deux types de diversification renvoient également à des principes de jugement du travail fondamentalement différents : le premier type s'appuie sur la vente à une clientèle " profane » qui valorise davantage la qualité de l'accueil, celle de produits «traditionnels » et un environnement « naturel» que la technicité et le rendement. Le second type s'appuie essentiellement sur un public de professionnels, de personnes « initiées » [Hughes, 1996], et donc sur des critères de jugement du travail qui restent dans le cadre de la profession [Paradeise, 1985].

Le premier type de diversification (ou de combinaison d'activités) peut sembler éloigné de la perception dominante du métier. Nous désignerons ces activités comme « en discontinuité ». Le second type de diversification au contraire reste conforme à la conception usuelle. Ce sont donc des activités « en continuité » par rapport au métier.

L'engagement de la conjointe sur l'exploitation (même combiné avec une activité professionnelle extérieure) semble donc favoriser l'émergence d'activités diversifiées en discontinuité avec le modèle professionnel, car ce sont des secteurs d'activité dont le chef d'exploitation ne veut pas se mêler. Les conjointes sont alors déléguées, voire reléguées à ce type de travail, peu intégré à l'exploitation. En revanche, les activités diversifiées en continuité avec le métier sont plus fréquentes pour les exploitations où la conjointe travaille à l'extérieur.

\section{Diversification et division conjugale du travail}

Nous allons vérifier maintenant que si la présence des femmes favorise la diversification en discontinuité, c'est bien parce que ce sont elles qui effectuent ces activités. Le recensement général agricole de 1988 fait une distinction entre activités agricoles et para-agricoles (ces dernières renvoient à la diversification structurelle) ${ }^{9}$ et donne les moyens de mesurer la quantité (exprimée en nombre d'unités de travail annuel ${ }^{10}$ ) de ces deux types de travail sur l'exploitation pour chaque membre de l'exploitation et la manière dont se répartit entre les conjoints le travail lié à telle ou telle activité diversifiée.

Les informations sur le temps de travail para-agricole des conjoints permettent de distinguer cinq types d'organisations conjugales du travail de diversification ou para-agricole : à côté de la majorité des exploitations qui ne déclarent pas de travail de diversification car celui-ci est trop occasionnel ou peu distinct du travail agricole ou domestique ${ }^{11}(59,8 \%$ des

164 A Travail, genre et sociétés n 30 - Novembre 2013 
cas), il y a celles où le travail para-agricole est effectué par des personnes autres que les deux membres du couple (1,3\%); celles où l'atelier est tenu seulement par le conjoint du chef d'exploitation $(12,1 \%)$; les «ateliers mixtes » où les deux conjoints travaillent dans l'atelier de diversification (7,2\%); enfin celles où ces activités sont faites par le seul chef d'exploitation $^{12}(19,6 \%)$.

Afin de faciliter les comparaisons, nous reprenons le même champ que pour les tableaux précédents (exploitations dont le chef est un homme vivant en couple) et nous nous concentrons sur les exploitations où le travail para-agricole est le plus important, soit 4787 exploitations.

Tableau 2 : Activités de diversification et organisation du travail para-agricole (en \% lignes)

\begin{tabular}{lccc}
\hline & $\begin{array}{c}\text { Atelier de } \\
\text { la conjointe }\end{array}$ & $\begin{array}{c}\text { Atelier } \\
\text { mixte }\end{array}$ & $\begin{array}{c}\text { Atelier } \\
\text { du chef }\end{array}$ \\
\hline Vente directe & $37,1 \%$ & $22,7 \%$ & $40,3 \%$ \\
\hline Vente produits bruts & $32,9 \%$ & $22,5 \%$ & $44,6 \%$ \\
\hline Vente produits transformés & $52,4 \%$ & $24,0 \%$ & $23,5 \%$ \\
\hline Accueil touristique & $48,6 \%$ & $22,0 \%$ & $29,4 \%$ \\
\hline $\begin{array}{l}\text { Ensemble des activités } \\
\text { en discontinuité }\end{array}$ & $39,6 \%$ & $22,0 \%$ & $38,5 \%$ \\
\hline Travaux agricoles à façon & $3,6 \%$ & $7,1 \%$ & $89,4 \%$ \\
\hline $\begin{array}{l}\text { Ensemble des activités } \\
\text { en continuité }\end{array}$ & $3,6 \%$ & $7,4 \%$ & $78,6 \%$ \\
\hline $\begin{array}{l}\text { Total des exploitations avec au moins } \\
\text { une activité de diversification }\end{array}$ & $32,4 \%$ & $18,6 \%$ & $49,0 \%$ \\
\hline
\end{tabular}

Source: Ministère de l'Agriculture, recensement général de l'agriculture 1988, échantillon au $1 / 10^{\mathrm{e}}$, exploitation secondaire.

Champ : Exploitations dont le chef est un homme vivant en couple, ayant une activité de diversification auquel l'un des membres du couple participe et pour laquelle un temps de travail est déclaré, $\mathrm{N}=4787$.

Lecture : $37,1 \%$ des exploitations qui ont une activité de vente directe ont un atelier tenu par leur conjointe.

Les activités de diversification en discontinuité et en continuité s'opposent à nouveau ici du point de vue de l'organisation du travail :

- les ateliers dont la conjointe est seule responsable et les ateliers mixtes ont tendance à être liés à des activités en discontinuité (vente directe, hébergement) ${ }^{13}$. La vente directe est cependant hétérogène : la vente de produits bruts ne semble pas liée à une division du travail particulière alors que la vente de produits transformés (foies gras, produits laitiers...) est organisée plus souvent que la vente de
${ }^{12}$ Pour toutes ces catégories, le chef ou sa conjointe peut être assisté dans son atelier d'un ou plusieurs autres membres de l'exploitation. Ce qui est testé avec cette variable c'est la division conjugale (et non pas familiale, ni sexuée) du travail de diversification.

\footnotetext{
${ }^{13}$ Plusieurs enquêtes de terrain sur la diversification confirment cette interprétation : la transformation et la vente de produits fermiers [Capt, 1991], la vente directe, l'hébergement et la restauration (en France, Danièle Capt [1994] ;

Christophe Giraud [2004] ; en Espagne, Gemma Cánoves, Maria Dolores GarcíaRamón et Nuria Valdovinos [1993] ; en Angleterre, Mary Bouquet [1985]) sont des activités où le travail et la responsabilité des femmes sont clairement mis en lumière.
} 
produits bruts sous la forme d'un atelier dirigé par la conjointe (54,42\% contre $32,9 \%)$.

- les ateliers tenus par le seul chef d'exploitation demeurent, eux, très fortement liés aux activités continues (travaux agricoles à façon, sylviculture...).

L'augmentation de la participation de la conjointe au travail agricole que nous avons constatée lorsque l'exploitation diversifie, renvoie donc à cette prise en charge de l'atelier para-agricole. La corrélation entre l'organisation du travail et les deux types de diversification est valable quelle que soit l'orientation productive des exploitations (cf. tableau 3 , en annexe). Si on peut dire que la diversification en discontinuité est une manière de résorber le sous-emploi féminin (l'autre résorberait le sous-emploi masculin), on peut ajouter que cette résorption ne se fait pas n'importe comment, puisque c'est surtout par la constitution d'ateliers indépendants: c'est l'autonomie qui est ici recherchée. Pour les activités en continuité, en revanche, le chef, responsable de l'atelier, n'associe pas sa femme mais peut éventuellement déléguer les tâches à un salarié.

Le contrôle direct, par les hommes chefs d'exploitation, des activités jugées stratégiques, qualifiées et qualifiantes, conduit les femmes à développer des ateliers qui sont dans les limbes de la reconnaissance professionnelle $(39,6 \%$ des activités en discontinuité sont effectuées par les conjointes seules contre 3,6\% des activités en continuité). Cependant, ce que les femmes trouvent dans ces activités, c'est la maîtrise d'un atelier autonome par rapport à leur mari. Cette maîtrise est une des revendications anciennes des femmes en agriculture [Barthez, 1982] : les groupements féminins n'ont pas revendiqué l'indépendance professionnelle des conjointes mais ont réaffirmé avec force le caractère conjugal des métiers de l'agriculture qui ne devait pas se confondre avec la soumission ou la dépendance des femmes envers leur mari. Ces espaces féminins d'autonomie sont notamment ceux qui correspondent aux activités diversifiées les moins légitimes aux yeux de la profession établie, mais c'est cette marginalité même qui fait qu'elles peuvent en être responsables. En s'appropriant des secteurs sans enjeu professionnel fort, elles obtiennent un territoire productif propre. Ce faisant, en s'ancrant dans des rôles qui sont dans le prolongement souvent des activités domestiques, elles renforcent davantage une certaine division sexuée du travail ou, à tout le moins, certains stéréotypes de sexe. En accueillant des touristes à la maison, elles jouent un rôle féminin qui n'intéresse pas les maris. Ainsi en s'appuyant sur et en renforçant les stéréotypes de sexe, les femmes peuvent construire des espaces d'autonomie qui leur permettent de réduire la rigueur des rapports sociaux de sexe. Car ces activités loin d'être une extension du domaine domestique 
Division conjugale du travail et légitimité professionnelle

sont des espaces où elles peuvent obtenir de leur propre travail une certaine valorisation sociale et économique.

\section{AUTONOMIE FÉMININE ET HÉTÉROGÉNÉITÉ DU MONDE AGRICOLE}

Le cœur du métier, malgré le vœu et le modèle proposé par Pierre Muller [Muller, Faure et Gerbaux, 1989], reste aujourd'hui centré sur la logique technicienne [Freidson, 1984] plutôt que sur des compétences d'articulation des activités, des compétences gestionnaires et la hiérarchisation des activités en agriculture perdure. Des activités en continuité avec le métier permettent aux chefs d'exploitation de conserver une image de professionnel et même d'élargir le champ de leurs compétences reconnues ${ }^{14}$; d'autres sans continuité, donc encore mal valorisées voire dévalorisées professionnellement, ou trop proches des tâches domestiques, sont investies par les conjointes.

Pour la diversification en continuité, ce sont les hommes exploitants qui, dans les secteurs modernistes comme dans d'autres moins sensibles au discours dominant, assurent ce type d'activité ou délèguent à des prestataires de service, des associés ou à des salariés. Ici, le collectif de travail est nonconjugal, la conjointe ayant souvent une carrière professionnelle propre extérieure à l'exploitation.

Cependant, le rôle de la conjointe est décisif dans le développement des activités diversifiées les moins reconnues professionnellement. Cet apport économique des femmes est souvent indispensable pour la préservation des exploitations petites ou moyennes et la qualité de vie des familles. Certains travaux ont pu conclure à un surcroit de domination : les femmes apporteraient un surtravail pour que l'exploitation dirigée par le mari puisse dégager les ressources nécessaires au budget de la famille [Cánoves, García-Ramón et Valdovinos, 1993]. On ne peut nier cependant que les ateliers de travail dirigés par les femmes changent très fortement la nature du travail féminin en agriculture : d'activité sans cesse entrecoupée, parcellisée, manquant de sens [Chabaud-Rychter, Fougeyrollas-Schwebel et Sonthonnax, 1985], il devient un travail plus contrôlé, réalisé dans la durée, avec des compétences spécifiques et que l'acteur peut s'approprier personnellement.

Les activités de diversification en discontinuité sont, pour les femmes qui en sont le plus souvent les responsables, des espaces de travail sur l'exploitation où elles acquièrent une certaine autonomie par rapport à leur mari, où elles rendent visibles leur travail et peuvent acquérir une forme de reconnaissance sociale et économique personnelle.

L'adoption de nouvelles activités dans le monde agricole dessine donc deux secteurs de ce monde déjà bien hétérogène, qui développent un rapport aux normes de la profession as-

\author{
${ }^{14}$ Dans le sens d'une \\ " écologisation du \\ métier d'agriculteur » \\ [Miéville-Ott, 2000] \\ tacitement acceptée par \\ la profession en \\ particulier dans les \\ zones en déprise, en \\ voie de reconnaissance \\ mais encore rarement \\ pleinement \\ revendiquée.
}


${ }^{15}$ Le nombre croissant d'exploitations où seul travaille le chef d'exploitation, parfois aidé(e) d'un salarié amène à considérer ces exploitations unipersonnelles comme des exploitations postfamiliales [Rémy, 2010]. sez différent. Le premier secteur regroupe des exploitations spécialisées et puissantes économiquement (céréaliculture) où le métier se complexifie par l'adjonction d'activités qui constituent le prolongement de l'activité centrale de l'exploitation. Les conjointes choisissent souvent d'avoir une activité professionnelle à l'extérieur de ces exploitations qui leur assure une forte autonomie ${ }^{15}$.

Le second secteur rassemble des exploitations moyennes et peu spécialisées (polyculture-élevage) qui restent attachées au modèle de l'agriculture conjugale mais où les nouvelles activités se développent sous la forme d'ateliers sans lien direct avec les compétences techniques légitimes du chef d'exploitation. La résistance aux rapports sociaux de sexe inégaux peut être lue à travers le développement de ces activités qui valorisent des compétences ou des rôles domestique féminins. En jouant sur les stéréotypes de sexe, les femmes travaillant sur l'exploitation agricole peuvent ainsi développer des activités économiques valorisantes, en rupture avec la logique technicienne de la profession, et disposer d'une autre forme d'autonomie [Singly, 1987].

Ainsi, depuis les travaux historiques conduits sur les femmes en agriculture ou au sein des ménages agricoles dans les années 1970, on est amené aujourd'hui à distinguer des offres plus diversifiées de professionnalisation féminine. En marge des deux secteurs que l'on vient d'expliciter, deux autres peuvent être dessinés : cela a été rapidement évoqué plus haut, certaines femmes, disposant ou non d'un conjoint, qui choisissent de s'installer elles-mêmes comme cheffes d'exploitation (le conjoint masculin ayant alors une activité non agricole) forment un troisième secteur. Toutes ne sont pas d'origine agricole. Les jeunes filles investissent de plus en plus les filières professionnelles agricoles au sein des lycées agricoles, refusant d'être cantonnées dans les sections de service, aux personnes par exemple [Dahache, 2012]. Le dernier secteur comporte des exploitations où les conjointes travaillent en partenariat avec leur compagnon au sein d'exploitations où elles peuvent rechercher une véritable reconnaissance de leurs compétences et un statut (celui d'associée ou de coexploitante), dans une activité partagée, comme par exemple la production laitière. En 2000, les exploitations où les conjointes se déclarent "aides familiales" sont, dans un tiers des cas, des exploitations diversifiées. Elles ne le sont que dans un quart des cas lorsque les conjointes se déclarent « exploitantes». La possession d'un statut reconnu sur l'exploitation inscrit ici davantage la conjointe dans la logique professionnelle technicienne que dans une logique de développement d'activités de diversification, ce qu'on peut comprendre comme un refus de se soumettre aux stéréotypes de sexe et une volonté d'affirmer pleinement un professionnalisme aussi grand que celui des exploitants masculins.

168 A Travail, genre et sociétés n 30 - Novembre 2013 
Division conjugale du travail et légitimité professionnelle

\section{BIBLIOGRAPHIE}

BARTHEZ Alice, 1982, Travail, famille et agriculture, Paris, Economica.

BARTHEZ Alice, 1986 «Du labeur paysan au métier d'agriculteur l'élaboration statistique en agriculture", Cahiers d'Economie et Sociologie Rurales, $\mathrm{n}^{\circ} 3$, pp. 46-72.

BERLAN Martine, 1982, "La gestion des aléas par les agricultrices", Pénélope : pour l'histoire des femmes, $\mathrm{n}^{\circ} 7$, pp. 71-73.

BESSIERE Céline, 2006, Maintenir une entreprise familiale. Enquête sur les exploitations viticoles de la région délimitée Cognac, Thèse de l'Université Paris Descartes.

BOUQUET Mary, 1985, Family, servants and visitors: the farmhousehold in nineteenth and twentieth century Devon, Norwich, Geobooks.

CÁNOVES Gemma, GARCíA-RAMÓN Maria-Dolores et VALDOVINOS Nuria, 1993, "Farm tourism, gender and the environment in Spain », Annals of Tourism Research, vol. 2, n³, pp. 267-282

CAPT Danièle, 1991, « Transformation, vente directe de produits fermiers et économies locales", Symposium international "Transformation des produits à la ferme », 7-9 mars, Paris.

CAPT Danièle, 1994, Demande de biens différenciés, comportements spatiaux et diversification de l'activité des exploitations agricoles, Rapport de recherche, INRA, Dijon, Université de Dijon.

CAPT Danièle et DUSSOL Anne-Marie, 2004, « Exploitations diversifiées : un contenu en emploi plus élevé ", Agreste Cahiers, n² 2, pp. 11-18.

CHABAUD-RYCHTER Danièle, FOUGEYROLlAS-SCHWEBEL Dominique et SONTHONNAX Françoise, 1985, Espace et Temps du travail domestique, Paris, Méridiens-Klincksieck.

DAHACHE Sabrina, 2010, «La singularité des femmes chef d'exploitation » in Bertrand HERVIEU, Nonna MAYER, Pierre MULlER, François PURSEIGLE et Jacques REMY (dir.), Les mondes agricoles en politique, Paris, Presses de Sciences Po, pp. 93-110.

DAHACHE Sabrina, 2012, La féminisation de l'enseignement agricole, Paris, L'Harmattan.

DELPHY Christine, 1983, «Agriculture et travail domestique : la réponse de la bergère à Engels ", Nouvelles Questions féministes, n 5, pp. 3-17.

DENTZER Marie-Thérèse, 1974, «Le rôle des conseillères ", Économie rurale, $n^{\circ}$ 99-100, «Le développement agricole en France », pp. 139-142.

FREIDSON Eliot, 1984, La profession médicale, Paris, Payot.

GARCIA-PARPET Marie-France, 2000, «Dispositions économiques et stratégies de reconversion. L'exemple de la nouvelle viticulture ", Ruralia, $\mathrm{n}^{\circ} 7$, mis en ligne le 22 janvier 2005 ,

<http:/ / ruralia.revues.org/document179.html>, consulté le 4 mars 2008.

GASSON Ruth, 1988, «Farm diversification and rural development », Journal of Agricultural Economics, vol. 39, n², pp. 175-182.

GIRAUD Christophe, 2001, Chambres d'hôtes à la ferme et autonomie de la femme en agriculture, Thèse de sociologie à l'Université Paris-Descartes.

GIRAUD Christophe, 2004, «Division du travail d'accueil et gratifications dans les chambres d'hôtes à la ferme », Cahiers du Genre, vol. 37, pp. 71-91.

Houssel Jean-Pierre, 1970, «Ville moyenne, développement de l'agriculture régionale, agriculture de banlieue: l'exemple de Roanne », Revue géographique de Lyon, vol. 45, n 4, pp. 391-408.

HUGHES Everett C., 1996, Le regard sociologique, Paris, Éditions de l'EHESS.

ILBERY Brian, 1991, "Farm diversification as an adjustment strategy on the urban fringe of the West Midlands », Journal of Rural Studies, vol. $7, \mathrm{n}^{\circ} 3$ pp. 207-218. 
LAGRAVE Rose-Marie (dir.), 1987, Celles de la terre : l'invention politique d'un métier, Paris, Éditions de l'EHESS.

LEMERY Bruno, 2003, "Les agriculteurs dans la fabrique d'une nouvelle agriculture », Sociologie du Travail, $\mathrm{n}^{\circ} 45$, pp. 9-25.

LERBOURG Jérôme, 2013, « Recensement agricole 2010. 12\% des exploitations développent une activité para-agricole », Agreste Primeur, n 302.

MARSDEN Terry K., MUNTON Richard J.C., WhATMORE Sarah J. et LitTLE Jo K., 1989, "Strategies for coping in capitalist agriculture: an examination of the responses of farm families in british agriculture ", Geoforum, n ${ }^{\circ}$, pp. 114.

MieVILLE-OTt Valérie, 2000, «Les éleveurs du Jura face à l'écologisation de leur métier », Le Courrier de l'Environnement, n 40, pp. 75-84.

MULLER Pierre, FAURE Alain et GerBAux Françoise, 1989, Les entrepreneurs ruraux, Paris, L'Harmattan.

PARADEISE Catherine, 1985, "Rhétorique professionnelle et expertise", Sociologie du Travail, vol. 27, $\mathrm{n}^{\circ}$ 1, pp. 17-31.

RATTIN Solange, 2002, «Les ménages d'agriculteurs en 2000 : un sur trois est pluriactif », Agreste Cahiers, n², pp. 3-14.

RATTIN Solange, 2006, "Actifs familiaux professionnels : les deux tiers sont des exploitants ", Agreste Cahiers, n 4, pp. 13-22.

REMY Jacques, 1987, « La crise de la professionnalisation en agriculture : les enjeux de la lutte pour le contrôle du titre d'agriculteur», Sociologie $d u$ Travail, vol.39, $\mathrm{n}^{\circ} 4$, pp. 415-441.

REMY Jacques, 2010, «Un monde en changement. Introduction » in Bertrand Hervieu, Nonna MaYer, Pierre Muller, François Purseigle et Jacques REMY (dir.), Les mondes agricoles en politique, Paris, Presses de Sciences Po, pp. 41-50.

SINGLY François DE, 1987, «Gagner sa place : la conquête de l'autonomie des femmes dans la famille ", Revue Internationale de l'Action Communautaire, $\mathrm{n}^{\circ} 18 / 58$, pp. $153-159$ 
Division conjugale du travail et légitimité professionnelle

\section{Annexe : L'adoption des activités diversifiées par les exploitations agricoles, une régression logistique}

L'analyse des deux formes de diversification (qui renvoie à deux rapports au métier et donc aussi à deux organisations du travail) doit faire intervenir les facteurs structurels classiques : secteur de production, taille économique de l'exploitation, âge des exploitants. En utilisant une régression logistique, nous avons pu vérifier, toutes choses égales par ailleurs, l'impact de l'organisation du travail et de l'engagement du conjoint du chef sur l'exploitation en prenant en compte l'influence des autres variables. Nous avons centré notre analyse sur les exploitations dont le chef d'exploitation vit en couple de manière à pouvoir approcher cette répartition conjugale du travail et nous avons pris en compte le sexe du chef d'exploitation dans la régression.

Tableau 3 : Diversification en continuité et en discontinuité et organisation du travail para-agricole

\begin{tabular}{|c|c|c|c|c|}
\hline & $\begin{array}{l}\text { Diversification } \\
\text { en discontinuité } \\
\text { (vente directe, } \\
\text { hébergement) }\end{array}$ & $\begin{array}{l}\text { Significa- } \\
\text { tivité }\end{array}$ & $\begin{array}{c}\text { Diversification } \\
\text { en continuité } \\
\text { (travaux à façon...) }\end{array}$ & $\begin{array}{l}\text { Significa- } \\
\text { tivité }\end{array}$ \\
\hline & $\mathrm{N}=12414$ & & $\mathrm{~N}=1365$ & \\
\hline Constante & 0,4720 & ** & $-4,8000$ & ** \\
\hline \multicolumn{5}{|c|}{ Temps de travail agricole du chef } \\
\hline Moins d'un mi-temps & - & ns & $-0,3026$ & $* *$ \\
\hline $\begin{array}{l}\text { D'un mi-temps à trois quarts } \\
\text { de temps }\end{array}$ & Référence & & Référence & \\
\hline Temps plein & $-0,2371$ & $* *$ & $-0,3054$ & ** \\
\hline \multicolumn{5}{|c|}{ L'organisation du travail para-agricole } \\
\hline $\begin{array}{l}\text { Pas de travail para-agricole } \\
\text { conjugal }\end{array}$ & $-2,1083$ & $* *$ & $-2,8971$ & $* *$ \\
\hline Atelier du seul conjoint & 0,8385 & $* *$ & $-0,7833$ & ** \\
\hline Atelier des deux conjoints & Référence & ** & Référence & \\
\hline Atelier du chef seul & $-1,1324$ & ** & 1,3929 & ** \\
\hline \multicolumn{5}{|c|}{ Engagement du conjoint par rapport à l'exploitation } \\
\hline Travail à l'extérieur & Référence & & Référence & \\
\hline $\begin{array}{l}\text { Travail à l'extérieur et } \\
\text { à l'intérieur }\end{array}$ & 0,2371 & $* *$ & 0,3996 & $* *$ \\
\hline Inactif partout & - & ns & - & ns \\
\hline $\begin{array}{l}\text { Travail à l'intérieur } \\
\text { moins d'un mi-temps }\end{array}$ & 0,0996 & * & 0,3268 & $* *$ \\
\hline $\begin{array}{l}\text { Travail à l'intérieur } \\
\text { un mi-temps et plus }\end{array}$ & 0,1508 & $* *$ & 0,2691 & $* *$ \\
\hline
\end{tabular}


Christophe Giraud et Jacques Rémy

\begin{tabular}{|c|c|c|c|c|}
\hline \multicolumn{5}{|l|}{ Âge du chef } \\
\hline Jeune (moins de 45) & $-0,2517$ & $* *$ & 0,4804 & $* *$ \\
\hline Âgé (45 et plus) & Référence & & Référence & \\
\hline \multicolumn{5}{|l|}{ Sexe du chef } \\
\hline Homme & - & ns & 1,3732 & $* *$ \\
\hline Femme & Référence & & Référence & \\
\hline \multicolumn{5}{|l|}{ Statut de l'exploitation } \\
\hline Individuelle & Référence & & Référence & \\
\hline Sociétaire & $-0,2664$ & $* *$ & 0,5197 & $* *$ \\
\hline \multicolumn{5}{|l|}{ Orientation productive } \\
\hline Céréales & $-0,1702$ & $* *$ & 1,4057 & $* *$ \\
\hline Maraîchage & 1,0198 & $* *$ & - & ns \\
\hline $\begin{array}{l}\text { Systèmes spécialisés (vignes, } \\
\text { fruits, lait, viande, granivores) }\end{array}$ & Référence & & Référence & \\
\hline \multirow[t]{6}{*}{$\begin{array}{l}\text { Systèmes complexes } \\
\text { (polyculture, polyélevage, } \\
\text { mixtes) }\end{array}$} & 0,5734 & $* *$ & 0,2657 & $* *$ \\
\hline & \multicolumn{4}{|c|}{$\begin{array}{l}\text { Modèle explicatif: modèle Logit expliquant la probabilité d'apparition dans les } \\
\text { exploitations agricoles d'un type d'activité de diversification. }\end{array}$} \\
\hline & \multicolumn{4}{|c|}{$\begin{array}{l}\text { Source: Ministère de l'Agriculture, recensement général de l'agriculture 1988, } \\
\text { échantillon au } 1 / 10^{\circ} \text {, exploitation secondaire. }\end{array}$} \\
\hline & \multicolumn{4}{|c|}{$\begin{array}{l}\text { Champ : exploitations avec conjoint, le chef étant un homme ou une femme, } \\
\mathrm{N}=55031 \text {. }\end{array}$} \\
\hline & \multicolumn{4}{|c|}{$\begin{array}{l}\text { Lecture : la probabilité qu'un coefficient soit non nul est, selon les cas, infé- } \\
\text { rieure à } 1 \%\left(\text { notée }{ }^{* *} \text { ) ou comprise entre } 1 \% \text { et } 5 \%\left(\text { noté }{ }^{*} \text { ) ou supérieure }\right.\right. \\
\text { (notée ns). Un coefficient positif indique une augmentation de la probabilité } \\
\text { de la modalité correspondante par rapport à la probabilité de la modalité de } \\
\text { référence alors qu'un coefficient négatif indique une baisse de cette même } \\
\text { probabilité. }\end{array}$} \\
\hline & \multicolumn{4}{|c|}{$\begin{array}{l}\text { Commentaire : Les deux régressions permettent de retrouver des résultats } \\
\text { connus dans la littérature : l'impact positif des secteurs céréaliers par rapport } \\
\text { aux autres systèmes spécialisés (vigne, fruits, lait et viande) sur le dévelop- } \\
\text { pement des travaux à façon (diversification en continuité). Les chefs d'exploi- } \\
\text { tation jeunes sont plus enclins à développer ce type d'activité que les chefs } \\
\text { plus âgés. De même les exploitations sociétaires développent ce type d'acti- } \\
\text { vités diversifiées plus souvent que les exploitations individuelles. Ces résul- } \\
\text { tats sont inversés quand on examine les activités en discontinuité. } \\
\text { Ces deux régressions permettent également de tester l'impact de l'organisa- } \\
\text { tion conjugale du travail dans l'atelier de diversification. Toutes choses égales } \\
\text { par ailleurs, les ateliers tenus par le conjoint seul favorisent fortement le } \\
\text { développement d'une activité diversifiée en discontinuité par rapport à un } \\
\text { atelier tenu par le seul chef d'exploitation. C'est l'inverse pour les activités en }\end{array}$} \\
\hline
\end{tabular}

172 A Travail, genre et sociétés n 30 - Novembre 2013 